\title{
Plastic Deformation Behavior and Mechanism of Bismuth Single Crystals in Principal Axes
}

\author{
Yuichi Yanaka ${ }^{1, *}$, Yoshiharu Kariya ${ }^{2, *}$, Hirohiko Watanabe ${ }^{3}$ and Hiroaki Hokazono ${ }^{3}$ \\ ${ }^{1}$ Graduate School of Shibaura Institute of Technology, Tokyo 135-8548, Japan \\ ${ }^{2}$ Department of Materials Science and Engineering, Shibaura Institute of Technology, Tokyo 135-8548, Japan \\ ${ }^{3}$ Fuji Electric Co., Ltd., Tokyo 191-0064, Japan
}

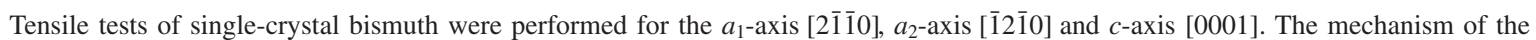
$a_{1}$-axis and $a_{2}$-axis plastic deformation at $298 \mathrm{~K}$ is twinning deformation at the initial deformation stage. Subsequent plastic deformation mechanism is the slip deformation that occurs by the changes in the crystallographic orientation inside twin crystals that is advantageous to the slip. The mechanism of the $c$-axis plastic deformation at $298 \mathrm{~K}$ is slip deformation, where the secondary slip system becomes activated. On the other hand, the slip deformation is the primary deformation mechanism and twinning deformation is not observed for any axes at $423 \mathrm{~K}$. [doi:10.2320/matertrans.MD201503]
\end{abstract}

(Received December 1, 2015; Accepted January 19, 2016; Published April 8, 2016)

Keywords: $\quad$ bismuth, single crystal, plastic deformation, tensile test, slip system, twin deformation

\section{Introduction}

In recent years, bismuth having a melting point of $544.7 \mathrm{~K}$ close to that of $\mathrm{Pb}$ is expected as a new promising base material alternative to lead-containing solder alloys. Especially, bismuth is attracting attention as a lead-free soldering material with a high melting point ${ }^{1-4)}$. In fact, however, mechanical characteristics and properties necessary for the reliability analysis of micro solder joints have seldom been investigated and they are still unknown. Bismuth is a semimetal that has metallic bonds and covalent bonds in its crystal structure. As shown in Fig. 1, the crystal structure of bismuth is a A7 (arsenic) type structure with the metallic bond layers and the covalent bond layers being laminated one after the other in the orientation of $[0001]^{5-7)}$. The slip system that can operate at ambient temperature is only $(0001)<\overline{1} \overline{1} 20>$ that does not cut covalent bonds. Therefore, plastic deformation by slip mechanism is difficult to occur ${ }^{8)}$. As for plastic deformation in bismuth, Motohashi et al. have investigated slip systems and twinning deformation using single crystals. The investigations, however, were performed under limited experimental

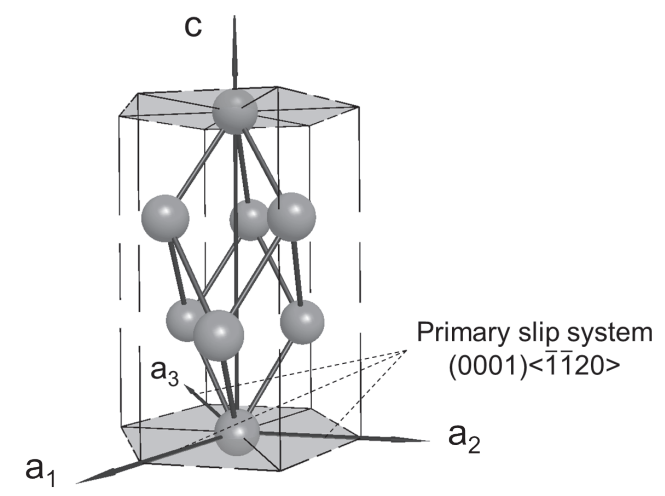

Fig. 1 Schematic illustration of crystal structure of bismuth.

*Corresponding author, E-mail: mb15039@ shibaura-it.ac.jp, y_kariya@ shibaura-it.ac.jp conditions. Thus, properties of plastic deformation required for the reliability assessment of solder joint structures have not been clarified yet ${ }^{9,10)}$.

In this study, a tensile testing was performed using bismuth of single-crystals to investigate stress-strain behavior in the principal axis.

\section{Experimental Procedure}

\subsection{Specimen preparation}

Discs of $99.999 \%$ purity bismuth were used in this study, which was grown to [0001] and [1100] (12 mm diameter and $1 \mathrm{~mm}$ thick for each) directions by the Bridgeman technique. The discs were cut out into strip specimens with tensile axes parallel to [2 $\overline{1} \overline{1} 0]\left(a_{1}\right.$-axis), [ $[\overline{1} 2 \overline{1} 0]\left(a_{2}\right.$-axis) and [0001] ( $c$-axis). For the cutting, a precision wire saw (K.D. Unipress) with a tungsten wire with $50 \mu \mathrm{m}$ in diameter was used. In order to observe slip lines, the prepared strip specimens were all mirror-finished by machine grinding/polishing. The grinding and polishing procedure was firstly grinding with $\mathrm{SiC}$ grinding paper (\#2400) followed by polishing with diamond particles $(6 \mu \mathrm{m} \rightarrow 3 \mu \mathrm{m} \rightarrow 1 \mu \mathrm{m})$ and lastly polishing with colloidal silica suspension. Crystallographic orientations of specimen for each axis are illustrated in Fig. 2. The $a_{1}$-axis, $a_{2}$-axis and $c$-axis specimens were in the orientation where the slip system hardly operates. Bismuth is liable to occur twinning deformation, so there is the potential of the deformation occurring during the fixation procedure of the specimens to a test machine. To avoid this, a brass chuck with grooves was preliminarily attached to the test machine as in the Fig. 3 and the strip specimens were bonded to the chuck by adhesives. UV curable adhesive (Henkel: Loctite 638UV) for testing at ambient temperature and inorganic adhesive (Toagosei: Aron Ceramic) for high temperature testing were used.

\subsection{Tensile test}

The test was done by the method of displacement control at test temperatures $298 \mathrm{~K}$ and $423 \mathrm{~K}$ and the initial strain rate 
$1 \times 10^{-3} / \mathrm{s}$. A micro-load mechnical testing machine with the adoption of a piezo actuator shown in Fig. 4 (Saginomiya: LMH207-20) was used. Due to constraints on the actuator strokes, the tensile test was not continued until crack occurred. However, the displacement of $400 \mu \mathrm{m}$ in the $298 \mathrm{~K}$ test and that of $200 \mu \mathrm{m}$ in the $423 \mathrm{~K}$ test were applied. Temperature was raised by the ceramic heater built in the fixing jig.

\subsection{Microstructural observation}

For observation of slip lines in slip deformation and changes in the surface configuration caused by twin crystals, motion videos were recorded using a stereomicroscope (Leica: Z16 APO) installed in the upper position of the test machine. And after the test, microstructures of the top surfaces and side surfaces of the specimens were observed by an optical microscope (Carl Zeiss: Axio Imager A1m). Then crystallographic
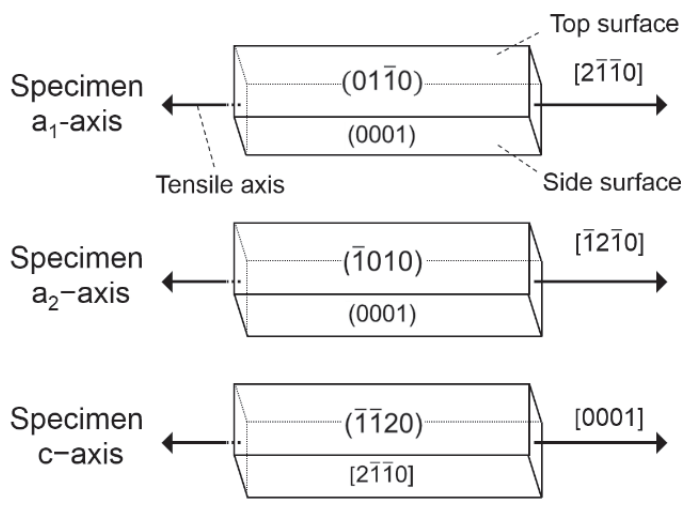

Fig. 2 Crystallographic orientation of loading axis and specimen surface.

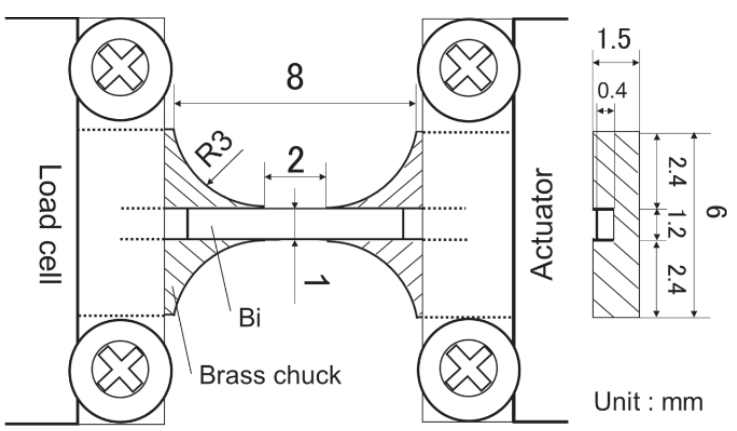

Fig. 3 Schematic illustration of specimen with fixing jig.

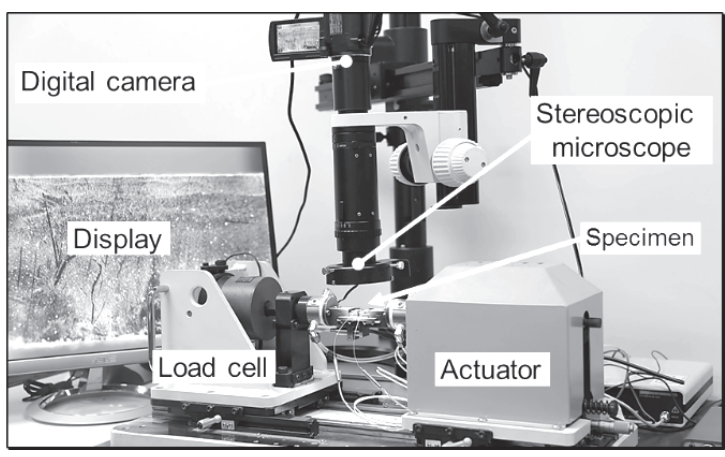

Fig. 4 Appearance of testing machine and in-situ observation system. orientation of the twins and slip system were determined. For the notation system for planes and directions in crystal lattices, the Miller-Bravais index that has four indices was used in this study ${ }^{5,11)}$.

\section{Results and Discussion}

\subsection{Plastic deformation behavior at $298 \mathrm{~K}$}

3.1.1 Stress-strain curve in each axial direction at $298 \mathrm{~K}$

The true stress-true strain curves in individual axis directions at $298 \mathrm{~K}$ are shown in Fig. 5. The stress-strain curves in the directions of the $a_{1}$-axis and the $a_{2}$-axis exhibited the behavior of progressive plastic deformation involving rapid stress decreases occurring several times immediately from the yielding point to the vicinity of $10 \%$ strain. After this stress oscillation, the curves showed a perfect plastic behavior with increased ductility. On the other hand, stress decreases observed on the $a_{1}$-axis were not confirmed in the stressstrain curve in the $c$-axis direction, where observed was a behavior in which deformation was progressing with concurrent moderate strain hardening. The yield stress was $13 \mathrm{MPa}$ at the $a_{1}$-axis, $15 \mathrm{MPa}$ at the $a_{2}$-axis and $12.5 \mathrm{MPa}$ at the $c$-axis. In any of these axial directions, no fracture occurred until $20 \%$ true strain.

\subsubsection{Deformation mechanism at $298 \mathrm{~K}$ in $a_{1}$-axis and $a_{2}$-axis}

As the slip system of bismuth that can operate at ambient temperature is only $(0001)<\overline{1} 120>$ of the primary slip system, twinning deformation easily occurs. A decrease in stress observed immediately after yielding was caused by twinning deformation. Images of top surface microstructures with $1 \%$ to $9 \%$ true strain obtained in situ observation during the testing are shown in Fig. 6. Twinning deformation was observed simultaneously with rapidly decreasing stress after the yielding. The orientation of the twin was determined to be (1102)[1101] known as the twin system of bismuth ${ }^{10,12-14)}$.

With deformation further progressing, slip lines were observed inside the twin crystals developed as can be seen in Fig. 6. The slip lines were generated by the activity of the slip system induced by changes in crystallographic orientation caused by twinning deformation. It has been reported that in

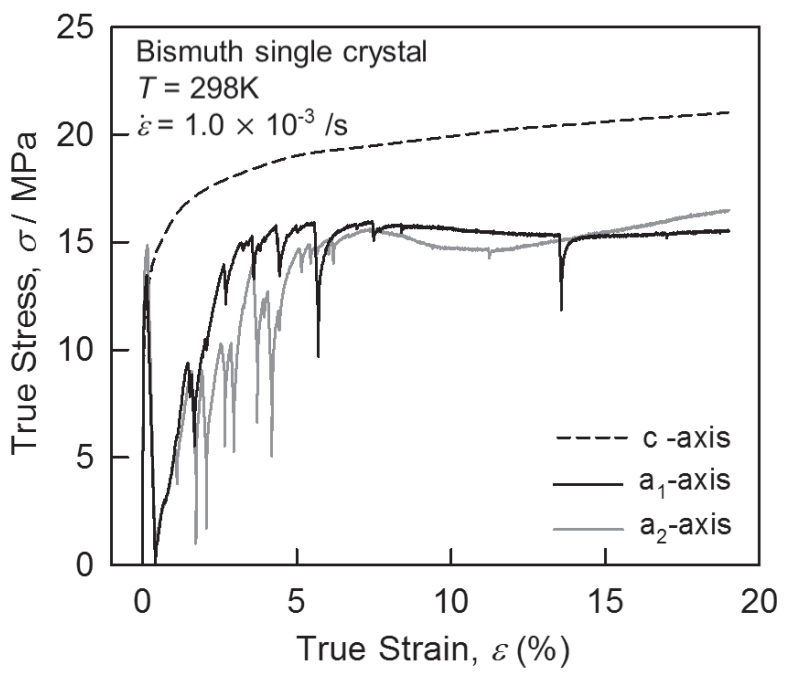

Fig. 5 Stress-strain curves for $a_{1}$-axis and $c$-axis at $298 \mathrm{~K}$. 


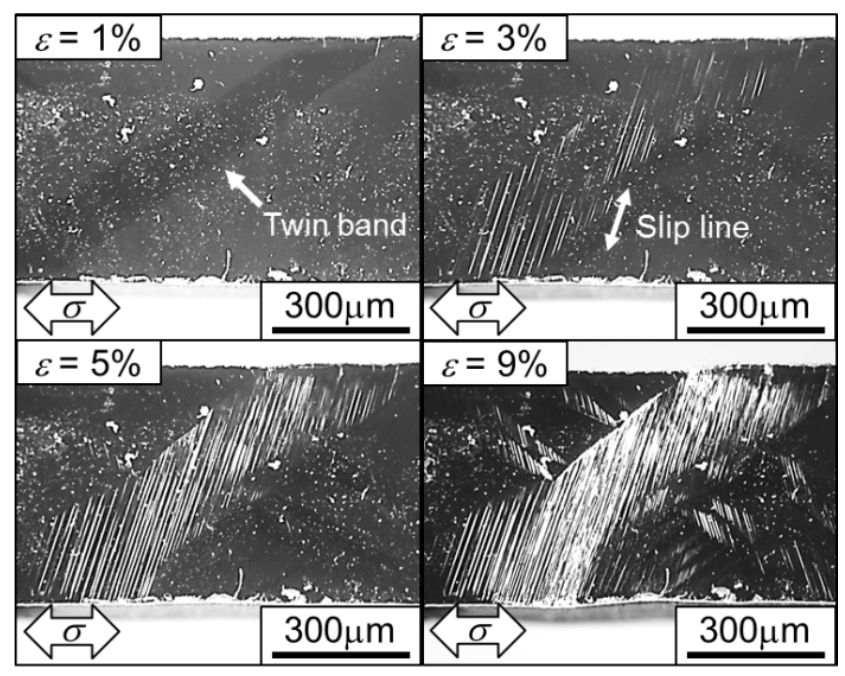

Fig. 6 Twin and slip band observed on the top surface of $a_{1}$-axis specimen during tensile test at $298 \mathrm{~K}$.

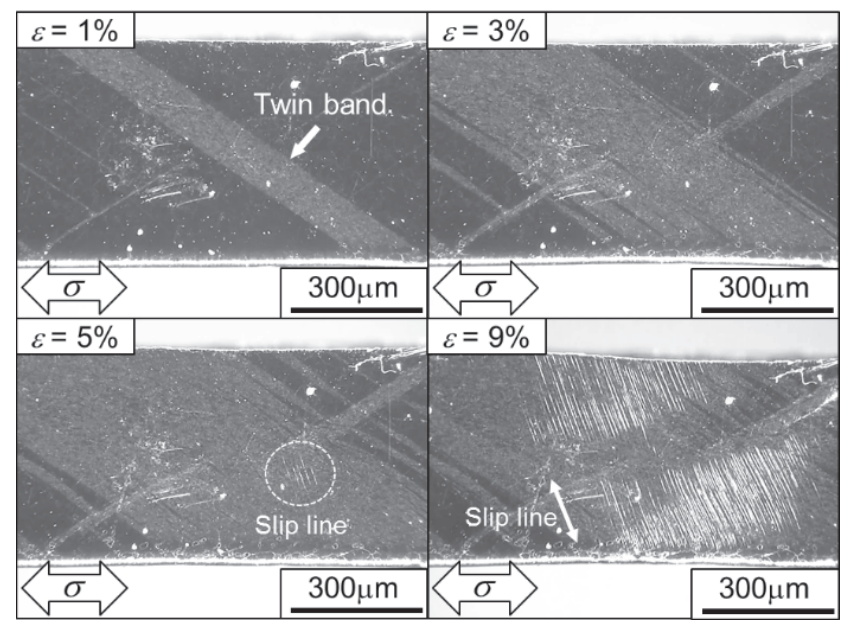

Fig. 7 Twin and slip band observed on the top surface of $a_{2}$-axis specimen during tensile test at $298 \mathrm{~K}$.

magnesium in which twin crystals develop, crystal rotation in the interior of twin crystals occurs when twin crystals are generated $^{15,16)}$. It seems that due to the crystal rotation, the crystallographic orientation inside twin crystals became advantageous to the activity of the primary slip system, compared to the initial orientation, resulting in slip deformation inside twin crystals. It has been also reported that single crystals of zinc and cadmium have the occurrence of slip deformation in their twin crystals. More detail of the mechanisms is not known yet ${ }^{17-19}$ ). As mentioned above, it has been demonstrated that bismuth has the deformation mechanism to gain ductility by twinning deformation and subsequent slip deformation inside twin crystals. There have been no reports showing that bismuth has deformation occurring in such a process. This is a new finding obtained by this study.

Top surface microstructures in situ observation with the true strain $1 \%$ to $9 \%$ in the $a_{2}$-axis direction during the testing are shown in Fig 7. On the $a_{2}$-axis, the twinning deformations were more clearly observed than on $a_{1}$-axis. And after twin crystals spreading over the entire specimen, the generation of slip lines was also observed. The observed slip lines were of

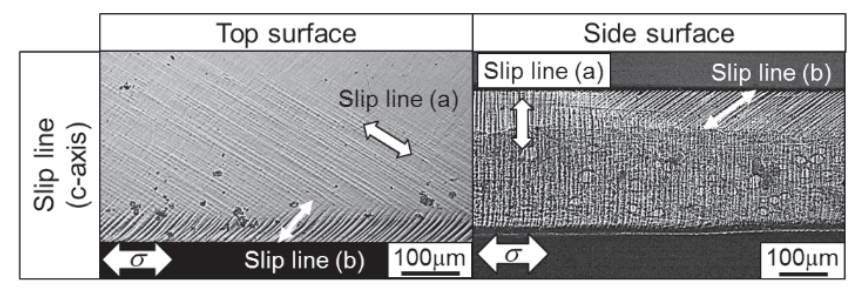

Fig. 8 Optical micrographs showing slip bands on the top surface and side surface of the specimen $c$-axis.

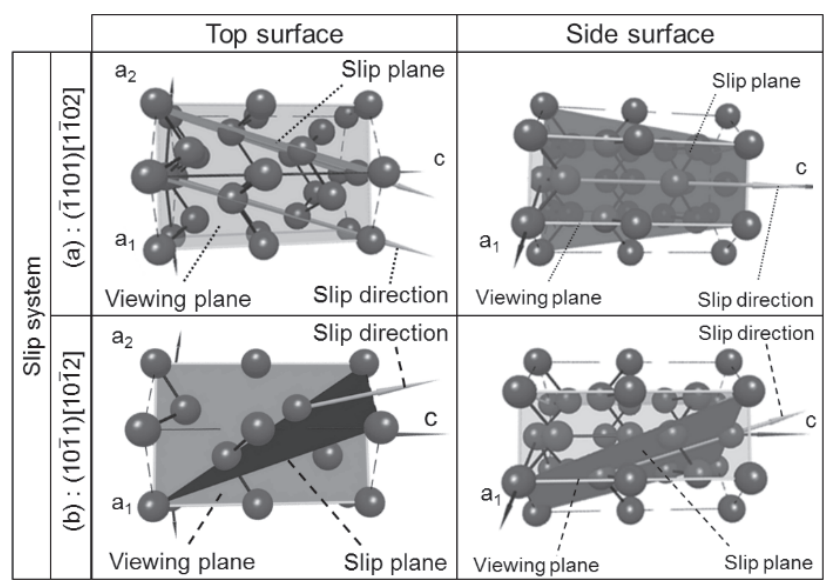

Fig. 9 Schematic illustration of $\{\overline{1} 101\}<1 \overline{1} 02>$ slip system.

(1102)[1]101] and the twinning deformation occurred on the same twin crystal surfaces as those observed in the results of $a_{1}$-axis. The reason of the differences in the surface morphology of $a_{1}$ and $a_{2}$ axes generated by twinning was not elucidated at the present time. A similar deformation mechanism appeared in both directions of the $a_{1}$-axis and the $a_{2}$-axis. This is because bismuth classified under the trigonal crystal system has the three-fold symmetry and the directions of the $a_{1}$-axis and the $a_{2}$-axis are equivalent with each other.

\subsubsection{Deformation mechanism in c-axis at $298 \mathrm{~K}$}

Figure 8 shows optical micrographs of the top surface and side surface of the $c$-axis specimen. As seen in Fig. 8, slip lines on the top surface and side surface of the specimen are visible. However, since the primary slip plane and the tensile axis are vertical to each other in the $c$-axis direction, the primary slip system has difficulty to get activated. The fact suggests that the slip lines observed in Fig. 8 occurred by the activity of the secondary slip system. Figure 9 shows a schematic illustration of the slip system (1101)[1102] and $(10 \overline{1} 1)[10 \overline{1} 2]$ viewed from the top surface and side surface. The slip lines seen in Fig. 8 corresponds to (1101)[1102] and $(10 \overline{1} 1)[10 \overline{1} 2]$. In this study, ( $\overline{1} 101)[1 \overline{1} 02]$ and $(10 \overline{1} 1)[10 \overline{1} 2]$ were operating for the $c$-axis specimen. It should be noted that for causing slip on $\{\overline{1} 101\}$, covalent bonds need to be cut, and $\{\overline{1} 101\}<1 \overline{1} 02>$ has difficulty getting activated and operating. However, compared to other atomic planes, the area density of $\{\overline{1} 101\}$ is higher and the distance between atomic layers is larger. In addition, the number of covalent bonds per unit area (i.e. the ratio of cutting covalent bonds in the case of slipping) is fewer compared to other planes, so it is thought that this slip system can be activated as the secondary slip 


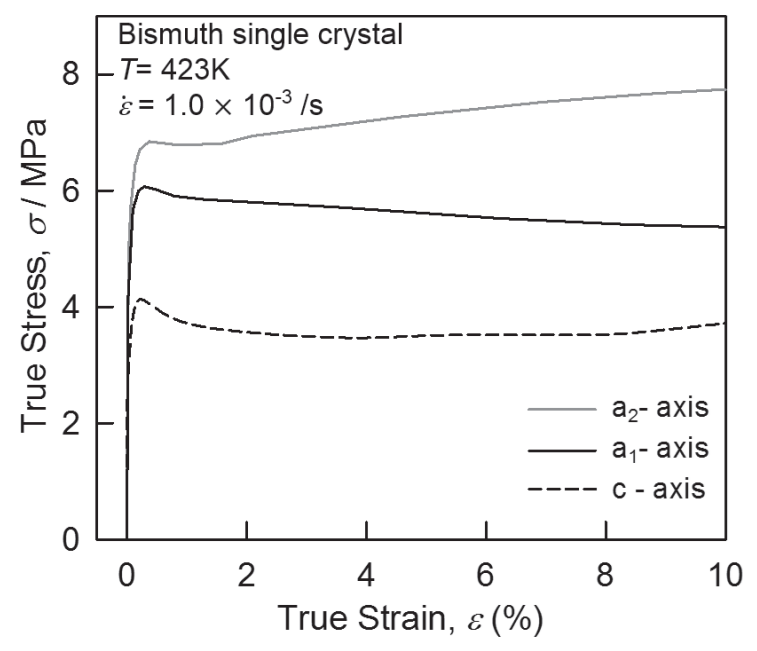

Fig. 10 Stress-strain curves for $a_{1}$-axis and $c$-axis tested at $423 \mathrm{~K}$.

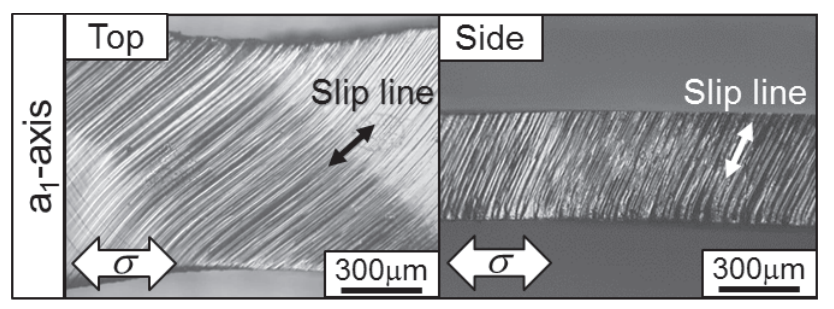

Fig. 11 Optical micrographs showing slip band of (22̄01) [ $\overline{1} \overline{1} 20]$ system on $a_{1}$-axis specimen tested at $423 \mathrm{~K}$.

system ${ }^{10)}$. From the above, as the $c$-axis has difficulty for the primary slip system to operate, bismuth in the $c$-axis obtains ductility by the activity of the slip system $\{\overline{1} 101\}<1 \overline{1} 02>$.

\subsection{Plastic deformation behavior and mechanism at $423 \mathrm{~K}$}

\subsubsection{Stress-Strain Curve at $423 \mathrm{~K}$}

Figure 10 shows the stress-strain curves in the directions of the $a_{1}$-axis, $a_{2}$-axis, and $c$-axis at $423 \mathrm{~K}$. It is confirmed that the deformation at $423 \mathrm{~K}$ was substantially different compared to $298 \mathrm{~K}$ in the directions of the $a_{1}$-axis and $a_{2}$-axis. On the $a_{1}$-axis, a decrease in stress due to twin crystals is not observed and only slip behavior is exhibited. In situ observation of the specimen surfaces, twin crystals were not present. The behavior of the $a_{2}$-axis was similar to that of $a_{1}$-axis with moderate strain hardening. On the $c$-axis, as at $298 \mathrm{~K}$, the behavior was slip deformation without strain hardening. Fracture did not occur in either of these directions until the end of the experiment. The yield stress was $6 \mathrm{MPa}, 7 \mathrm{MPa}$, and $4 \mathrm{MPa}$ on the $a_{1}$-axis, $a_{2}$-axis and $c$-axis, respectively. The yield stress at $423 \mathrm{~K}$ decreases to roughly $1 / 2$ on the $a_{1}$-axis and $a_{2}$-axis and to $1 / 3$ on $c$-axis at $298 \mathrm{~K}$, respectively.

\subsubsection{Deformation mechanism of the $a_{1}$-axis and $a_{2}$-axis at $423 \mathrm{~K}$}

The $a_{1}$-axis at $423 \mathrm{~K}$ exhibited only a slip behavior without twinning deformation - this seems to be caused by an increase in the number of active slip systems which was increased by an increase in temperature; thereby the slip system inactive at ambient temperature operated. Figure 11 shows

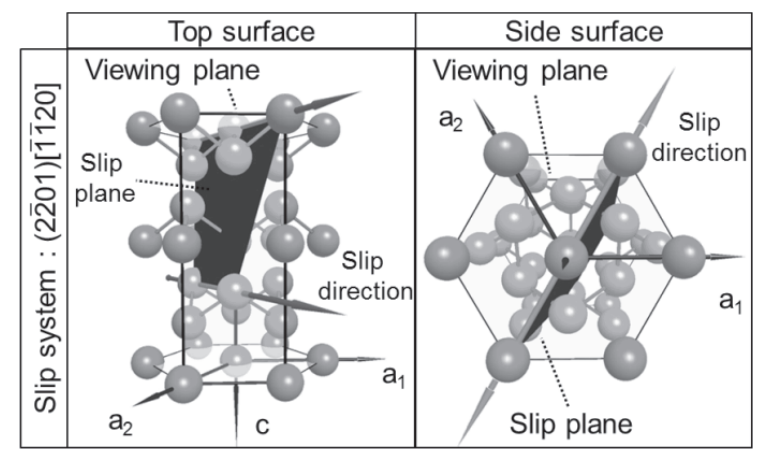

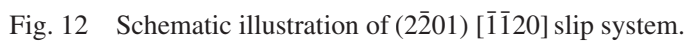

Table 1 Relationship between schmid factor of each slip systems and the number of covalent bonds included per unit area, $B^{10)}$.

\begin{tabular}{c|c|c|c}
\hline \hline Tensile direction & Most severely stressed slip systems & Schmid factor & $B$ \\
\hline \multirow{4}{*}[11\overline{2}0]{} & $(0001)[2 \overline{1} \overline{1} 0]$ & 0.000 & 0.00 \\
\cline { 2 - 4 } & $(\overline{1} 101)[\overline{1} 10 \overline{2}]$ & 0.343 & 2.00 \\
\cline { 2 - 4 } & $(\overline{1} 101)[11 \overline{2} 0]$ & 0.354 & 2.00 \\
\cline { 2 - 4 } & $(2 \overline{2} 01)[\overline{1} \overline{1} 20]$ & 0.416 & 2.28 \\
\hline
\end{tabular}

the surface of the $a_{1}$-axis specimen tested. Although there are no twin crystals on the surface, slip lines are uniformly seen on the entire specimen. The slip system that was activated can

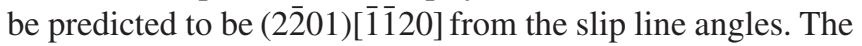
schematic illustration of this slip system is shown in Fig. 12. The slip line angles well matches up with those in Fig. 11. This slip system had been pointed out as a slip system, other than the primary slip system, that has the potential of being activated. However, there has been no report to date that the foregoing slip system that has a high density of covalent bonds on the slip plane has ever operated in uniaxial test-

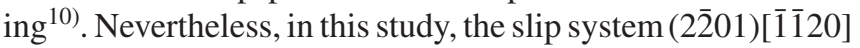
was activated and operated by elevating temperature. It is predicted that the activity of slip system in bismuth is not easily affected by covalent bonding due to an increase in temperature. Table 1 shows the relationship between individual slip systems, Schmid factors, and the number of covalent bonds per unit area when [1120] equivalence to the $a_{1}$-axis is the tensile axis. As seen in Table 1, the value of the Schmid factor of $(2 \overline{2} 01)[\overline{1} \overline{1} 20]$ that operated in this study is the largest, indicating that the activity of the slip system at $423 \mathrm{~K}$ is governed only by the Schmid factor, and that whether the slip systems can operate with ease or not is non-dependent on the number of covalent bonds on the slip plane. That is, it is predicated that at $423 \mathrm{~K}$, the Peierls barrier of the slip systems including covalent bonds is lowered and the differences in critical resolved shear stresses for any slip systems have already disappeared. According to the pressure-temperature phase diagram of bismuth, the phase at high temperature/pressure transits from semimetal to metal, while the phase does not shift to metal under normal pressure ${ }^{20,21)}$. This means that at $423 \mathrm{~K}$ under normal pressure, a phase shift to metal does not occurs but covalent bonds exist inside the crystals. It is clear, however, that at $423 \mathrm{~K}$ the slip system including covalent bonds that 


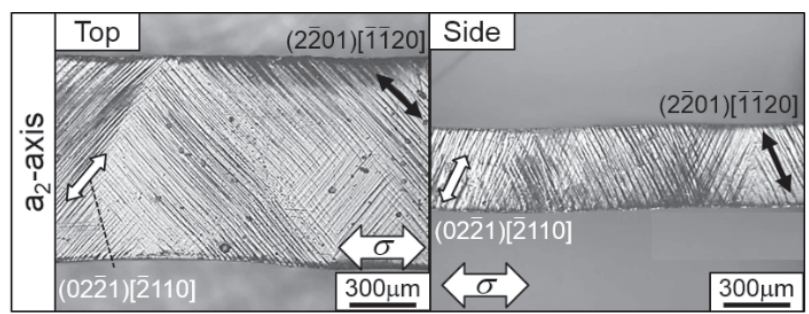

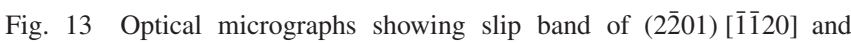
(02) 21 ) $[\overline{2} 110]$ systems on $a_{2}$-axis specimen tested at $423 \mathrm{~K}$

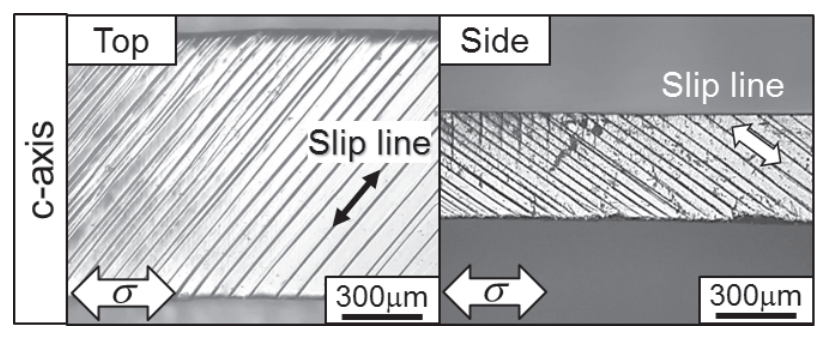

Fig. 14 Optical micrographs showing slip band of (0111) [01리 system on $c$-axis specimen tested at $423 \mathrm{~K}$.

have difficulty being activated have operated, suggesting that there is the potential of some type of changes occurring in covalent bonds due to an increase in temperature.

The surface of the $a_{2}$-axis specimen tested is shown in Fig. 13. Twin crystals on the surface are not observed in Fig 13. Unlike the $a_{1}$-axis, two slip systems were operated on the $a_{2}$-axis. The strain hardening seen in the stress-strain curve of the $a_{2}$-axis seems to have been caused by double slip. However, it is unclear why two slip systems were active in the $a_{2}$-axis at the present time. From the slip line angles, the slip systems that operated were $(2 \overline{2} 01)[\overline{1} \overline{1} 20]$ and $(02 \overline{2} 1)[\overline{2} 110]$. These slip systems were equivalent to the slip system that operated on the $a_{1}$-axis. The ease of slip deformation seems to become non-dependent on the number of covalent bonds on slip plane by increasing temperature. From the foregoing, in the plastic deformation of the $a_{2}$-axis at $423 \mathrm{~K}$, twinning deformation was not observed as in the case of $a_{1}$-axis, and the two slip systems that were inactive at ambient temperature acted.

\subsubsection{Deformation mechanism of $c$-axis at $423 \mathrm{~K}$}

The top surface and side surface of the $c$-axis specimen tested are shown in Fig. 14. As seen in Fig. 14, slip lines on the top surface and side surface of the $c$-axis specimen at $423 \mathrm{~K}$ tested were uniformly observed. These slip lines are clearer than those observed at $298 \mathrm{~K}$, revealing that in bismuth, slip deformation is easily caused by an increase in temperature. The slip system that was operated was $(0 \overline{1} 11)$ [011 2 ], which was in an equivalent relation with $\{1101\}<1 \overline{1} 02>$ that was operated at $298 \mathrm{~K}$. Thus, the secondary slip system of $\{\overline{1} 101\}<1 \overline{1} 02>$ independent from temperature is active in the $c$-axis direction. And it is considered that the yielding stress was reduced at $423 \mathrm{~K}$, since the critical resolved shear stress decreases with increasing test temperature.

\section{Conclusion}

A tensile test was conducted for the $a_{1}$-axis [2 $\left.\overline{1} \overline{1} 0\right], a_{2}$-axis [1 $2 \overline{1} 0]$ and $c$-axis [0001] in the principal axis orientation using sing-crystal bismuth to investigate the plastic deformation behavior and deformation mechanisms. Findings are as follows:

(1) The mechanism of the $a_{1}$-axis and $a_{2}$-axis plastic deformation at $298 \mathrm{~K}$ is twinning deformation at the initial deformation stage. Subsequent plastic deformation mechanism is the slip deformation that occurs by the changes in the crystallographic orientation inside twin crystals that is advantageous to the slip.

(2) The mechanism of the $c$-axis plastic deformation at $298 \mathrm{~K}$ is slip deformation, where $\{\overline{1} 101\}<1 \overline{1} 02>$ of the secondary slip system becomes activated.

(3) In the $a_{1}$-axis and $a_{2}$-axis plastic deformation at $423 \mathrm{~K}$, twinning deformation is not observed and slip deformation is the primary deformation mechanism.

(4) In this deformation, $(2 \overline{2} 01)[\overline{1} 120]$ that have a high number of covalent bonds on slip surfaces is activated. And in the $a_{2}$-axis plastic deformation, double slips by

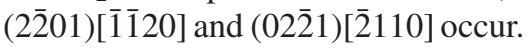

(5) The $c$-axis plastic deformation at $423 \mathrm{~K}$ is the same slip deformation as that occurring at ambient temperature, and regardless of temperature, $\{\overline{1} 101\}<1 \overline{1} 02>$ is activated.

\section{REFERENCES}

1) V. Chidambaram, J. Hattel and J. Hald: Microelectron. Eng. 88 (2011) 981-989.

2) K. Suganuma, S.J. Kim and K.S. Kim: JOM J. Min. Met. Mat. Soc. 61 (2009) 64-71.

3) Y. Takaku, I. Ohnuma, R. Kainuma, Y. Yamada, Y. Yagi, Y. Nishibe and K. Ishida: J. Electron. Mater. 35 (2006) 1926-1932.

4) J.N. Lalena, N.F. Dean and M.W. Weiser: J. Electron. Mater. 31 (2002) 1244-1249.

5) P. Hofmann: Prog. Surf. Sci. 81 (2006) 191-245.

6) R.J. Needs, R.M. Martin and O.H. Nielsen: Phys. Rev. B 33 (1986) 3778-3784.

7) Ralph W. G. Wyckoff: CRYSTAL STRUCTURES, (Inter-science Publishers, New York, 1963) pp. 32-33.

8) R. E. Slonaker and M. Smutz: Factors affecting the growth and the mechanical and physical properties of bismuth single crystals, (Ames, Iowa, Ames Laboratory, 1964) pp. 56-61.

9) S. Otake and Y. Motohashi: J. Japan Inst. Metals 37 (1973) 44-51.

10) Y. Motohashi and S. Otake: J. Japan Inst. Metals 37 (1973) 978-986.

11) B. D. Cullity: Elements of X-ray diffraction, (Addison-Wesley Publishing Company, Inc, U. S., 1956) pp. 462-463.

12) V.I. Bashmakov and T.S. Chikova: Crystallogr. Rep. 47 (2002) 489494.

13) O.M. Ostrikov: Tech. Phys. Lett. 46 (2001) 1199-1201.

14) A.I. Pinchuk and S.D. Shavrei: Phys. Solid State 43 (2001) 39-41.

15) H. Yoshinaga and R. Horiuchi: Trans. Jpn. Inst. Met. 4 (1963) 1-8.

16) J. Koike: Metall. Mater. Trans., A Phys. Metall. Mater. Sci. 36 (2005) 1689-1696.

17) R.L. Bell and R.W. Cahn: Proc. R. Soc. Lond. A Math. Phys. Sci. 239 (1957) 494-521.

18) T. Nakajima, I. Fujishiro, K. Kurita and H. Mii: J. Soc. Mater. Sci., JPN. 20 (1971) 962-967.

19) T. Nakajima, I. Fujishiro and S. Muto: J. Soc. Mater. Sci., JPN. 36 (1987) 847-853.

20) W. Klement, Jr., A. Jayaraman and G.C. Kennedy: Phys. Rev. 131 (1963) 632-637.

21) F.P. Bundy: Phys. Rev. 110 (1958) 314-318. 